\title{
Effects of miR-155 on High Glucose Induced- Cardiac Developmental Defects of Zebrafish Embryos
}

\section{Kai Zhang}

Nanjing Medical University

Yijian Zhang

Nanjing Medical University

Mengyuan Huang

Nanjing Medical University

Xiangjiang Yuan

Nanjing Medical University

Liyuan Wei

Nanjing Medical University

Deli Li

Nanjing Medical University

Yan Zhang

Nanjing Medical University

Hongjiao Liang

Nanjing Medical University

\section{Lintao Wang}

Nanjing Medical University

Xuejun Wang

Nanjing Medical University

Bai Jin

Nanjing Medical University

Zhengxian Tao

Nanjing Medical University

Li Yuan ( $\nabla$ yuanli@njmu.edu.cn )

Nanjing Medical University https://orcid.org/0000-0002-0284-5854

Original investigation

Keywords: Ets1, miR-155, Igf1, heart development, congenital heart disease, diabetes mellitus, zebrafish embryos, ROS 
Posted Date: August 4th, 2020

DOl: https://doi.org/10.21203/rs.3.rs-42391/v1

License: (c) (i) This work is licensed under a Creative Commons Attribution 4.0 International License. Read Full License 


\section{Abstract}

Background: Gestational diabetes mellitus (GDM) is known to have a teratogenic effect on heart development. However, the underlying mechanisms are still unclear. Former studies determined that miR155 is elevated in GDM patients. Besides, miR-155 is a key molecule for development. In the present study, we explored the potential role of miR-155 in heart development and the effect of miR-155 on high glucose-induced cardiac developmental defects.

Methods: Zebrafish embryos were exposed to $2 \%$ D-Glucose in a fluctuating manner. Activators or inhibitors of miR-155, Ets1, and Igf1 were injected into one-cell stage embryos. The expression levels of miR-155, Ets 1 and cardiac specific genes were evaluated by real-time PCR. The regulation of Igf1 by Ets 1 was examined using luciferase assays. The levels of reactive oxygen species (ROS) were analyzed by DCFH-DA. Proteins involved in Igf1 pathway were detected by western blot analysis. Maternal serum miR155 was determined using ELISA. Fetal cardiac structural and functional characteristics in diabetic or healthy pregnancies were performed by echocardiography.

Results: miR-155 levels are increased in serum from GDM patients and are correlated with fetal cardiac structural changes. High glucose exposure in zebrafish embryos altered the morphology of the heart, impaired the heart function, and increased the expression of miR-155 as well as cardiac specific genes. Upregulation of miR-155 activated Igf1-Akt-Gsk3 $\beta$ pathway by targeting Ets 1 and increased the production of ROS and may thereby exert teratogenic effect on cardiac development. In addition, knockdown of miR-155 blocked Igf1 survival pathway and induced apoptosis and may thus induced zebrafish cardiac developmental defects.

Conclusion: miR-155 is a key molecule for heart development and is involved in high glucose-induced cardiac malformation, and it might be a novel biomarker as well as a potential drug target of high glucose-induced cardiac defects.

\section{Background}

Diabetes mellitus (DM) is characterized by high levels of blood glucose. During pregnancy, DM is correlated with an elevated risk for congenital heart disease (CHDs), e.g., atrial or ventricular septal defects, Tetralogy of Fallot and transposition of the great arteries, in the offspring of humans and animals [1-3]. The potential teratogenic effect of high glucose on heart morphogenesis has been documented in previous research [4]; however, the mechanism remains unclear.

miR-155 is a well-known multifunctional microRNA that participates in diverse physiology and pathology processes including inflammation, metabolism, and tumor genesis $[5,6]$. Recently, several studies reported that miR-155 also functions as a key molecular in development. For example, Burocchi et al [7] determined that miR-155 directly targets Ets proto-oncogene 1 (Ets1) to modulate human T cells development. Interestingly, previous studies identified that both miR-155 and Ets 1 are also expressed in cardiomyocytes $[8,9]$. Besides, it has been demonstrated that Ets1 plays a vital role in myocardial 
development in chicken embryos [10]. Therefore, it would be interesting to explore whether miR-155 could regulate heart development via targeting Ets1. Besides, former study reported that miR-155 is upregulated in the heart of diabetic mice[8]. Moreover, circulating miR-155 is elevated in gestational DM (GDM) patients [11]. Considering the effect of high glucose on cardiovascular system and miR-155, and the potential role of miR-155 in heart development, we consequently hypothesized that miR-155 may be involved in high glucose-induced cardiac developmental defects.

In mammalian models, such as mice and rats, the observation of heart development can only be made through successive sections and immunohistochemical staining. These methods can not reflect the dynamic real-time development changes of heart [12]. Zebrafish is an outstanding model organism for studying development.[13]. Its rapid lifecycle, high productivity and transparent developing embryos make it easier to study dynamic changes in vivo which are not readily achievable in other animal models [14]. Moreover, the resting blood glucose levels of zebrafish embryos as well as adult zebrafish are very close to humans $[15,16]$. Here, we investigated the effects of miR-155 on high glucose-induced cardiac developmental defects in zebrafish embryos. In addition, we detected the serum miR-155 of healthy and diabetic pregnancies and analyzed its correlation with cardiac structural and functional characteristics of their offspring.

\section{Methods}

\section{Zebrafish usage and maintenance}

Zebrafish maintenance and procedures were conducted in accordance with the medical ethics committee of Nanjing Medical University. All experiments were approved by the medical ethics committee of Nanjing Medical University. Wild-type Zebrafish and Cmlc2:GFP transgenic zebrafish were obtained from the Institute Of Hydrobiology, Chinese Academy Of Sciences (Nanjing, China). Normally developing embryos were selected and raised in embryonic medium, also referred as $\mathrm{E} 3$ medium $(5 \mathrm{mM} \mathrm{NaCl}, 0.17 \mathrm{mM} \mathrm{KCl}$, $0.33 \mathrm{mM} \mathrm{CaCl}_{2}$, and $0.33 \mathrm{mM} \mathrm{MgSO}_{4}$ ), under standard conditions at $28.5^{\circ} \mathrm{C}$ with a $14 \mathrm{~h}$ light $/ 10 \mathrm{~h}$ dark cycle according to standard procedures.

\section{Human subjects}

We enrolled 43 patients that were diagnosed with GDM (according to guidelines of the American Diabetes Association in 2013) and treated from January 2017 to December 2018 in the Department of Obstetricians and Gynecologists, Jiangsu province Hospital. 20 of them showed poorly controlled blood glucose (according to the treatment targets recommended in the guidelines of American Diabetes Association, fasting blood glucose $>5.5 \mathrm{mmol} / \mathrm{L}$ and $2 \mathrm{~h}$ postprandial blood glucose $>6.7 \mathrm{mmol} / \mathrm{L}$ were regarded as uncontrolled). Patients with one or more exclusion criteria according to the previous study were excluded [17] . In addition, we recruited 25 gestational ages-matched healthy pregnant women as controls. Fetal echocardiography was performed in these female participants at 35 weeks using 
VOLUSON 730 ultrasound systems (GE Medical Systems Kretztechnik GmbH \& Co. OHG). All participants were informed about the study and a written informed consent was provided by each participant.

\section{Treatment of zebrafish embryos with high glucose}

To simulate blood sugar in a fluctuating manner, the environment of zebrafish embryos was alternated every $24 \mathrm{~h}$ between $2 \%$ D-glucose and E3 media until 72 hour post-fertilization (hpf) using a methodology modified previous study [18]. Control embryos were only maintained in E3 media.

\section{Treatment of zebrafish embryos with N-acetylcysteine (NAC)}

NAC was dissolved in dimethyl sulphoxide (DMSO) at stock concentrations of $10 \mathrm{mM}$. After treatment with high glucose or injection with miR-155 mimics at $3 \mathrm{hpf}$, zebrafish embryos were treated with $100 \mu \mathrm{M}$ NAC until 72hpf. Control embryos were treated with the equivalent amount of DMSO solution. Final concentration of DMSO is less than $0.1 \%$ and had no obvious side-effect on zebrafish embryonic development. Each treatment group was replicated three times using embryos obtained from three independent spawns.

\section{Western blot analysis}

For western blot analyses, embryos at $36 \mathrm{hpf}$ were collected and then were lysed in RIPA lysis buffer and proteinase inhibitor mixture. The concentration of the protein was analyzed by bicinchoninic acid (BCA) method. Protein samples were separated via 10\% SDS-PAGE and transferred to the PVDF (polyvinylidene difluoride) membranes for $100 \mathrm{~min}$ at the $100 \mathrm{~V}$. The membranes were blocked with $5 \%$ non fat dry milk and then incubated with the appropriate primary antibodies at $4{ }^{\circ} \mathrm{C}$ overnight, followed by incubation with the corresponding secondary antibodies. Antibodies against phospho (p)-Akt (Ser473) (\#4060), Akt (\#4685) were purchased from Cell Signaling Technology. Ets1 (\#4685) was purchased from Santa Cruz biotechnology. Igf1 (\#DF 6096), Bax(\#AF0120), Bcl2 (\#AF6139), p-GSK3ß (Ser 9) (\#AF2016), GSK3 $\beta(\# A F 5016)$ were purchased from Affinity Biosciences.

\section{Total RNA extraction and RT-PCR}

Total RNA from whole embryos at $72 \mathrm{hpf}$ was extracted using TRIzol reagent according to the manufacturer's protocol (Invitrogen, Shanghai, China). cDNA was synthesized by the PrimeScript ${ }^{\mathrm{TM}}$ reverse transcript reagent Kit (Takara, Tokyo, Japan). The QPCR reactions were prepared with SYBR Green Master (Roche), and then run 35 cycles of $32^{\circ} \mathrm{C}$ for $15 \mathrm{~min}, 42^{\circ} \mathrm{C}$ for $15 \mathrm{~min}, 72^{\circ} \mathrm{C}$ for $5 \mathrm{~min}$, and $4^{\circ} \mathrm{C}$ for 5 min by real-time PCR detect system (Bio-Rad, Hercules, USA). Relative gene expression levels were calculated using the $2^{-\triangle \Delta C t}$ method, with $18 \mathrm{~S}$ used as the reference gene and normalized. PCR primer sequences were synthesized by Sangon Biotech Co., Ltd (Shanghai, China) and listed in Supplementary Table. S1.

\section{Reverse transcription and qRT-PCR for miR-155}


Stem-loop RT-PCR was performed as previously described [19]. Briefly, miR-155 and U6 specific cDNAs were synthesized with TaqMan MicroRNA Reverse Transcription Kit, according to the manufacturer's protocol. The Bulge-loop ${ }^{\mathrm{TM}}$ miRNA qRT-PCR Primer specific for dre-miR-155 and U6 is designed by RiboBio (Guangzhou, China). The qPCR reactions were mentioned above. The PCR primers were listed as follows: for U6, forward primer 5'- TTGGTCTGATCTGGCACATATAC -3' and reverse primer 5'AAAAATATGGAGCGCTTCACG -3'; for miR-155, forward primer 5'- CGCCGTTAATGCTAATCGTGATAG -3' and reverse primer 5'- GCAGGGTCCGAGGTATTCCG -3'. Relative miR-155 expression levels were calculated using the $2^{-\triangle \Delta C t}$ method, with $U 6$ used as the reference gene and normalized.

\section{Detection of intracellular ROS}

ROS activities were measured at 24 hpf using an ROS assay kit (Nanjing Jiancheng Bioengineering Institute, China). Briefly, $20 \mu \mathrm{M}$ of DCFH-DA (2,7-dichlorofurescin diacetate) was added to the culture medium containing the embryos to be tested. After incubation at a constant temperature $\left(28.5^{\circ} \mathrm{C}\right)$ for 1.5 $\mathrm{h}$, the medium was discarded and PBS was used to wash the embryos for three times. After washing with PBS for three times, embryos were lysed in RIPA lysis buffer and centrifuged at $12,000 \mathrm{~g}$ for $1 \mathrm{~min}$ at $4{ }^{\circ} \mathrm{C}$. Finally, the supernatants were collected for detecting the activities of ROS at the excitation wavelength of $500 \pm 15 \mathrm{~nm}$ and emission wavelength of $530 \pm 20 \mathrm{~nm}$. The fluorescence values were normalized to protein content for each group.

\section{Measurement of miR-155 levels in maternal serum}

Blood samples were collected from all participants upon their admission. Serum was immediately prepared by centrifugation at $4^{\circ} \mathrm{C}, 3000 \mathrm{~g}, 10 \mathrm{~min}$, and then stored at $-80^{\circ} \mathrm{C}$ for subsequent use. Serum miR-155 levels were detected using a commercial double-sandwich enzyme-linked immunosorbent assay (ELISA) according to the manufacturer's instructions (Shanghai Enzyme-linked Biotechnology Co., Ltd, Shanghai, China). The dynamic range of miR-155 detection varies from 38 to $1400 \mathrm{pg} / \mathrm{mL}$. miR-155 levels were determined using standard curves, reading the optical density at $450 \mathrm{~nm}$ on an automatic plate reader (Biotek, Winooski, USA). All samples were run in duplicate, and concentration values were averaged.

\section{Plasmids construction}

The DNA fragment containing the zebrafish Ets1 or Igf1 promoter was amplified by PCR from zebrafish genomic DNA using the Pyrobest PCR kit (Takara, Tokyo, Japan). The PCR products were then subcloned into the C1al and Xbal sites of the pCS2 ${ }^{+}$vector (Shanghai Qin cheng Biotechnology Co., Ltd, Shanghai, China) to construct the pCS2 ${ }^{+}$-Ets 1 plasmid and pCS2 ${ }^{+}$-Igf1 plasmid or into the $\mathrm{Kpnl}$ and Sacl sites of pGL3-promoter vector (Promega, USA) to construct the pGL3-basic-zebrafish-Igf1 promoter plasmid. Besides, pGL3-mutant-zebrafish-Igf1 promoter plasmid was also constructed, which contained Ets1binding site mutant in Igf 1 promoter. The integrity of these plasmids was confirmed by sequencing. The primers are listed in Supplementary Table 2. 


\section{Luciferase reporter assay}

Dual-luciferase reporter assay to the analysis of promoter activity in zebrafish embryos was performed as previously described [20]. Briefly, $80 \mathrm{pg}$ of corresponding Igf1 luciferase reporter DNA and $10 \mathrm{pg}$ of Renilla DNA were injected either alone or together with $50 \mathrm{pg}$ of Ets $1 \mathrm{mRNA}$ into one-cell stage embryos. Injected embryos were incubated for $12 \mathrm{~h}$ at $28.5^{\circ} \mathrm{C}$ in egg water and then were dissolved in passive lysis buffer for luciferase assays. Luciferase activities were measured using a dual-luciferase reporter assay kit (Promega, USA). The firefly luciferase activity was normalized using the Renilla luciferase activity levels.

\section{Microinjection}

Microinjection was performed using back-filled fine borosilicate glass capillary needles. Plasmids, mRNA and morpholinos (MOs) were injected into the cytoplasm of the embryos. The detail of microinjection has been described previously [21] . MOs against Ets1 or Ets2 were obtained from Gene Tools (Corvallis, OR). The morpholinos sequences are as follows: Control MO, 5'-CCTCTTACCTCAGTTACAATTTATA-3'; Ets1 MO, 5'-GATGACTTACGTCTACTTTTTCGGA-3'; Igf1 MO, 5'-GAAGAAATGACCGCTAGACATCCCT-3'. Activator and inhibitor of dre-miR-155 were purchased from RiboBio (Guangzhou, China). The dre-miR-155 mimic and inhibitor sequences are as follows: dre-miR-155 mimic, forward 5'- UUAAUGCUAAUCGUGAUAGGGG-3', reverse 5'- CCCCUAUCACGAUUAGCAUUAA-3'; dre-miR-155 inhibitor, 5'- CCCCUAUCACGAUUAGCAUUAA-3'. Besides, for mRNA injections, plasmids of $\mathrm{pCS} 2^{+}$-Ets 1 and $\mathrm{pCS} 2^{+}$-lgf1 were linearized with $\mathrm{C} 1$ al. Capped mRNA for microinjection was synthesized with SP6 mMessage mMachineTM kit (Ambion, Thebarton, SA, Australia).

\section{Statistical analysis}

Experiments were conducted at least three times independently. SPSS software (Version 21, SPSS Inc., Chicago, IL, USA) was utilized for the statistical analyses. Dates were expressed as mean \pm SD.

Significance of differences among groups was evaluated by Student's t-test. Spearman correlation was used to analyze relation of maternal serum miR-155 levels to fetal cardiac ventricular walls thicknesses. Values of $P<0.05$ were considered to be statistically significant.

\section{Results}

\section{Serum miR-155 levels were increased in GDM patients and correlated with foetal ventricular walls thicknesses}

To begin with, we detected the levels of miR-155 in maternal serum and analyzed its correlation with offspring. The general clinical data gathered for each group is presented in Table 1. Compared to healthy group, the BMI, FBG and $2 \mathrm{~h}$ OGTT in GDM groups were higher $(P<0.01)$. Besides, the foetal interventricular septal thickness (IVST), right ventricular wall thickness (RVWT), left ventricular wall thickness (LVWT) and birth weight were higher in the diabetic groups than those in non-diabetics $(P<0.05)$. Moreover, as shown in Fig. 1a, serum levels of miR-155 were significantly increased in GDM 
groups $(P<0.01)$. The concentration of miR-155 in pregnant women showed positive correlation with fetal IVST $(r=0.295, P<0.05)$, LVWT $(r=0.297, P<0.05)$ and RVWT $(r=0.248, P<0.05)$ (Fig. 1b-d). These observations suggested that serum miR-155 levels were increased in GDM patients and associated with foetal ventricular walls thicknesses.

Table 1

Clinical characteristics of study participants

\begin{tabular}{|c|c|c|c|}
\hline Characteristics & $\begin{array}{l}\text { Health subjects }(n= \\
25)\end{array}$ & $\begin{array}{l}\text { Controlled GDM(n = } \\
\text { 23) }\end{array}$ & $\begin{array}{l}\text { Uncontrolled GDI } \\
\text { 20) }\end{array}$ \\
\hline \multicolumn{4}{|c|}{ Maternal information } \\
\hline Age (years) & $26.51 \pm 4.17$ & $28.72 \pm 4.55$ & $28.80 \pm 3.86$ \\
\hline Delivery weeks & $39.13 \pm 1.59$ & $38.45 \pm 1.92$ & $38.42 \pm 1.85$ \\
\hline BMI $\left(\mathrm{kg} / \mathrm{m}^{2}\right)$ & $26.36 \pm 2.87$ & $29.33 \pm 3.61^{\star \star}$ & $32.32 \pm 3.29^{* \star \# \#}$ \\
\hline FBG (mM) & $4.49 \pm 0.27$ & $5.07 \pm 0.33^{\star \star}$ & $5.69 \pm 0.22^{\star \star}, \# \#$ \\
\hline 2 h OGTT (mM) & $4.89 \pm 0.50$ & $6.06 \pm 0.41^{\star *}$ & $8.24 \pm 0.99^{* \star}, \# \#$ \\
\hline
\end{tabular}

Newborn information

\begin{tabular}{|c|c|c|c|}
\hline Birth weight (g) & $3205.35 \pm 392.73$ & $3516.97 \pm 485.30^{*}$ & $3637.54 \pm 570.97^{\star \star}$ \\
\hline IVSD thickness (mm) & $2.97 \pm 0.65$ & $3.34 \pm 0.44^{*}$ & $3.74 \pm 0.54^{* *, \#}$ \\
\hline $\begin{array}{l}\text { RVWD thickness } \\
(\mathrm{mm})\end{array}$ & $2.62 \pm 0.40$ & $2.94 \pm 0.37^{\star \star}$ & $3.10 \pm 0.42^{\star \star}$ \\
\hline $\begin{array}{l}\text { LVWD thickness } \\
(\mathrm{mm})\end{array}$ & $2.74 \pm 0.29$ & $3.07 \pm 0.62^{*}$ & $3.30 \pm 0.53^{\star \star}$ \\
\hline LVEF (\%) & $64.48 \pm 3.60$ & $63.13 \pm 3.15$ & $63.40 \pm 3.21$ \\
\hline Heart rate (bpm) & $134.18 \pm 7.63$ & $132.42 \pm 11.07$ & $129.65 \pm 15.22$ \\
\hline
\end{tabular}

HS: health subjects, GDM: gestational diabetes mellitus, BMI: body mass index, FBG: fasting blood glucose, OGTT: oral glucose tolerance test, IVSD: interventricular septal thickness at end diastole, RVWD: right ventricular wall thickness at end diastole, LVWD: left ventricular wall thickness at end diastole, LVEF: left ventricular ejection fraction

Values were presented as mean \pm SD. $* P<0.05$, $* * P<0.05$ vs corresponding health subjects. $\# P<0.05$, $\# \# P<0.05$ vs corresponding controlled GDM.

\section{Knockdown of miR-155 can rescue high glucose-induced zebrafish cardiac developmental defects}

Blood sugar levels are normally maintained within a narrow range; however, this precise physiological mechanism is disordered in women with pre-existing or gestational diabetes [15]. To simulate blood 
sugar in a fluctuating manner, the environment of zebrafish embryos was alternated every $24 \mathrm{~h}$ between normal E3 medium and 2\% D-glucose. High glucose exposures in zebrafish embryos altered embryonic heart morphology, caused pericardial edema, impaired heart rate and increased the expression of cardiac related genes (Fig. 1f-h). In addition, the levels of miR-155 were significantly increased in high glucosetreated embryos (Fig. 1e). Interestingly, the deformed phenotypes, decreased heart rate and abnormally expressed cardiac related genes were, at least partially, rescued when miR-155 inhibitors were treated (Fig. 1f-h). These results indicated that miR-155 may be involved in high glucose-mediated heart developmental defects in zebrafish embryos.

\section{Overexpression of miR-155 causes zebrafish heart developmental defects}

Considering miR-155 was up-regulated in high glucose-treated zebrafish embryos and was involved in high glucose-induced zebrafish cardiac developmental defects, we therefore supposed that overexpression of miR-155 may also impair zebrafish heart development. To confirm this, $3 \mu \mathrm{M}$ of miR155 mimics were injected into zebrafish embryos. As shown in Fig 2a,c-d, overexpression of miR-155 resulted in cardiac morphology and functional defects, and increased the expression of cardiac related genes. Besides, we used the Cmlc2:GFP zebrafish to investigate the heart from fertilized eggs to the completely developed process. As shown in Fig 2b, overexpression of miR-155 caused chamber abnormalities, increased ventricular wall thickness and disorganized layers. Interestingly, the impaired heart morphology and function, and abnormal expression of cardiac specific markers were almost completely rescued when miR-155 mimics were injected together with miR-155 inhibitors (Fig 2). These results confirmed that miR-155 is essential for heart development, and up-regulation of miR-155 could cause zebrafish cardiac developmental defects.

\section{miR-155 regulates zebrafish cardiac development by targeting Ets 1}

Previous studies demonstrated that the region of the 3' UTR of mouse and human Ets 1 containing the conserved miR-155 binding site[22]. Using bioinformatics analysis, we found that zebrafish Ets 1 gene also has at least one potential binding site at its 3'-UTR for miR-155 (Supplementary Fig S1). Given Ets1 plays a vital role in cardiac development, we were interested in exploring whether or not miR-155 mediates high glucose-induced zebrafish cardiac developmental defects by targeting Ets1. As shown in Fig 3b, high glucose declined the expression of Ets1, while knockdown of miR-155 could attenuate such abnormal decrease. Besides, overexpression of Ets1 was able to rescue high glucose-mediated zebrafish heart developmental defects and reduced the abnormal increase of cardiac gene expression (data not shown). In addition, overexpression of Ets 1 could reverse cardiac developmental defects in miR-155 mimics-injected embryos (Fig 2). Moreover, knockdown of Ets1 resulted in the same abnormal phenotypes as overexpression of miR-155 did in zebrafish embryos, while downregulation of miR-155 could rescue Ets1 knockdown-caused cardiac developmental defects (Fig 3a, c-d). Taken together, these results determined that Ets 1 is the downstream target gene of miR-155 in zebrafish embryos, and miR155 could mediate high glucose-induced zebrafish cardiac developmental defects by targeting Ets 1 .

\section{miR-155 regulates zebrafish cardiac development through Igf1 signaling}


The above results showed that miR-155 was able to regulate zebrafish heart development by targeting Ets1; however, the potential downstream signal transduction pathways remain unclear. Using bioinformatics analysis, we found that Igf1 is probably a downstream of the transcription factor Ets 1 . To confirm this, luciferase assay was conducted. As shown in Fig 4b, overexpression of Ets 1 markedly attenuated luciferase levels in pGL3-basic-zebrafish-Igf1-promoter plasmid -injected embryos, while had no effect on pGL3-mutant-zebrafish-Igf1 promoter plasmid-injected embryos (pGL3-mutant-zebrafish-lgf1 promoter plasmid containing Ets1-binding site mutant in Igf1 promoter). Besides, knockdown of Ets1 dramatically increased the expression of Igf 1 (Fig. 3c). These results confirmed that Igf1 is the downstream target of Ets1 and Ets1 exerts a negative effect on the expression of Igf1 in zebrafish embryos. Moreover, overexpression of Igf1 resulted in the same phenotype as upregulation of miR-155 or downregulation of Ets1 did in zebrafish embryos (Fig 4a,c-d). Knockdown of miR-155 or overexpression of Ets 1 could rescue cardiac defects in Igf1 mRNA-injected embryos (Fig 4a,c-d). Above all, these results suggested that miR-155 regulates zebrafish heart development through Igf1 signaling.

\section{Upregulation of miR-155 activated Igf1-Akt-Gsk3 $\beta$ pathway in zebrafish embryos}

Previous studies determined that Igf1-Akt-Gsk3 $\beta$ pathway plays a vital role in the process of myocardial hypertrophy $[23,24]$. In the current study, we found that overexpression of miR-155 increased the levels of proteins (p-Akt, p-Gsk3 $\beta$ ) involved in Igf1 pathway and knockdown of miR-155 and Igf1, or overexpression of Ets1 could reverse these abnormal increases (Fig 4e,g). In addition, the protein levels of p-Akt and pGsk3 $\beta$ were also markedly increased in high glucose-treated zebrafish embryos, and it is conceivable that knockdown of miR-155 or Igf1, or overexpression of Ets1 could reduce such abnormal increase (Fig 4f,h). These results indicated that under high glucose condition, upregulation of miR-155 could activate Igf 1 pathway and may thereby cause zebrafish heart developmental defects.

\section{Upregulation of miR-155 elevated the production of ROS in zebrafish embryos.}

Previous study reported that reactive oxygen species (ROS) accumulation were partially reversed in miR155 knockout mesenchymal stem cells [25]. Besides, plenty of researches determined that abnormal increase of ROS leads to developmental defects $[26,27]$. In the current study, DCFH-DA was used to explore the levels of ROS in high glucose-treated zebrafish embryos. As shown in Fig $5 b$, high glucose increased the production of ROS, while such abnormal increase were partially prevented when miR-155 inhibitor was treated. Besides, NAC is a well-known ROS inhibitor. It could reduce the abnormal increase of ROS caused by high glucose (Fig $5 \mathrm{~b}$ ). In addition, NAC had no influence on the expression of miR-155 (Fig $5 e$ ). Although NAC could not reverse abnormal increase of cardiac gene expression (data not shown), it alleviated pericardial edema, improved heart rate in high glucose-treated zebrafish embryos (Fig $5 a, c)$. Similarly, overexpression of miR-155 also elevated the production of ROS (Fig 5f). Overexpression of Ets1 or knockdown of Igf1 or treatment of NAC could reduce the abnormal increase of ROS (Fig 5f) and rescue the deformed phenotypes (Fig $5 \mathrm{~d}, \mathrm{~g}$ ). These results suggested that under high glucose condition, upregulation of miR-155 increased the production of ROS in zebrafish embryos and elevated ROS could exert teratogenic effect on zebrafish heart development. 


\section{Knockdown of miR-155 can also induce cardiac developmental defects in zebrafish embryos}

According to the above results, upregulation of miR-155 could impair cardiac development and function. In the next study, we were interested in identifying whether downregulation of miR-155 affects the development of heart. We injected $5 \mu \mathrm{M}$ of miR-155 inhibitor into wild type as well as Cmlc2:GFP transgenic zebrafish embryos and found that downregulation of miR-155 interfered with normal cardiogenesis, caused cardiac looping defects, declined overall heart size, decreased the expression of cardiac related genes, and impaired embryonic heart function (Fig 6a-d). As expected, overexpression of Ets1 or knockdown of Igf1 resulted in the same phenotype as downregulation of miR-155 did (Supplementary Fig S2, Supplementary Fig S3). Besides, previous study determined that Bcl2 is a downstream of Igf1 and inhibits apoptosis in many cell types $[28,29]$. In the current study, we found that knockdown of miR-155 significantly decreased the proteins levels of $\mathrm{Bcl} 2$ and markedly increased the Bax protein level, while these changes were attenuated by Ets1 MO or Igf1 mRNA treatment (Fig 6e). Above all, these results suggested that downregulation of miR-155 blocked Igf1 survival pathway, inducing apoptosis and may thereby cause cardiac developmental defects in zebrafish embryos.

\section{Discussion}

In the present study, we have made three major findings. First, miR-155 levels are increased in serum from GDM patients and are correlated with fetal cardiac structural changes. Second, high glucose elevated the expression of miR-155 in zebrafish embryos. Upregulation of miR-155 activated Igf1-Akt-Gsk3 $\beta$ pathway as well as increased the production of ROS and may thereby exert teratogenic effect on cardiac development (Supplementary Fig S4). Third, knockdown of miR-155 inhibited Igf1 survival pathway and induced apoptosis and may thus hinder zebrafish embryonic and cardiac development (Supplementary Fig S4). These findings not only revealed the role of miR-155 in heart development, but also illustrated the effects of miR-155 on high glucose-induced cardiac developmental defects and highlighted the clinical potential of miR-155 as biomarkers for diagnosis and drug target of GDM-induced CHDs.

Congenital cardiovascular malformations form the most prevalent group of birth defects and about one in every 100-200 babies is born with a heart defect [30]. These defects can occur for many different reasons including genetic factors, exposure to environmental teratogens and birth injury. Diabetes in pregnant women is known to have a teratogenic effect on the development of fetal cardiovascular system [31]. In the present study, we found that foetal ventricular walls thicknesses were thicker in diabetic groups. Although the potential mechanisms for the correlation between GDM and fetal heart defects remain unclear, some evidences have proved that high glucose plays a vital role in the development of fetal cardiac anomalies [32]. In the current study, D-Glucose exposure in zebrafish embryos elevated the overall heart size and increased the expression of cardiac related genes, while Lglucose did not have such effect on zebrafish embryos development, suggesting that these abnormal phenotypes are not due to the osmotic effect. Interestingly, knockdown of miR-155 can rescue high glucose-induced zebrafish cardiac developmental defects. Moreover, overexpression of miR-155 caused the same abnormal phenotypes as high glucose exposure did in zebrafish embryos. Some possible 
explanations may illustrate these results. Firstly, in the current study, we identified that miR-155 is involved in zebrafish heart development. Under high glucose condition, the expression of miR-155 was elevated and mediated high glucose-induced heart developmental defects. Secondly, we determined that miR-155 could regulate cardiac development through Igf1 pathway. It has been reported that Igf1 is crucial for cardiomyocyte proliferation during zebrafish heart development. Besides, Igf1 could cause cardiomyocyte hypertrophy via activation of Gsk3 $\beta$ phosphorylation $[23,24]$. In the current study, we found that upregulation of miR-155 activated Igf1-Akt-Gsk3 $\beta$ pathway and may thus cause cardiac developmental defects.

Recently, several studies reported that high glucose could increase the production of ROS in cardiomyocytes [33,34]. Given a number of researches reported that abnormal increase of ROS could cause developmental defects, it would be interesting to explore whether high glucose could increase the production of ROS and may thereby exert teratogenic effect on cardiac development $[26,27]$. In the current study, we found that high glucose could increase ROS production in zebrafish embryos and cause heart developmental defects, while these deformed phenotypes and abnormal increase of ROS could be reduced in the presence of NAC or miR-155 inhibitor. These results could be explained by the following statement: To begin with, it has been demonstrated that upregulation of miR-155 increases ROS production via suppressing antioxidation-related genes expression in mesenchymal stem cells [25]. In the present study, we found that high glucose elevated the expression of miR-155 and may thus enhance the production of ROS. Knockdown of miR-155 could reverse such abnormal increase. Furthermore, NAC is a well-known ROS scavenger. It could scavenge excess ROS and may thereby rescue the deformed phenotypes. In addition, we also found that overexpression of Ets 1 or knockdown of Igf 1 could reduce the production of ROS in miR-155 mimics-injected zebrafish embryos. Reasons below may illustrate these discoveries: Initially, it has been demonstrated that Ets 1 can regulate the expression and activity of intracellular glutathione peroxidase (GPX) expression that acts as a free radical scavenger [35]. Downregulation of Ets1 may decrease the glutathione levels and thus increase intracellular ROS. Additionally, former study suggested that Igf1 could increase midgut mitochondrial ROS synthesis [36]. Similarly, exogenous Igf1 is able to induce ROS production in mouse cortical collecting duct cells [37]. Above all, it may be possible that high glucose increased the expression of miR-155 and upregulation of miR-155 declined the expression of Ets 1 and elevated the levels of Igf1 resulting in the abnormal increase in the production of ROS and may thereby cause zebrafish embryonic and cardiac defects.

In the current study, we also explored whether downregulation of miR-155 affects the development of heart and found that knockdown of miR-155 caused cardiac looping defects, decreased the expression of cardiac related genes and overall size of heart, and impaired embryonic heart function. We listed three possible reasons: Firstly, knockdown of miR-155 declined the levels of Igf1 and may thereby inhibit cardiomyocyte proliferation during zebrafish heart development [38]. Secondly, Bcl2 is a downstream of Igf1 and could inhibit apoptosis in many cell types $[28,29]$. In the current study, we found that knockdown of miR-155 reduced the expression of Bcl2 but markedly increased the levels of Bax. Thirdly, previous study has proved that a decrease in Gsk3 $\beta$ phosphorylation on Ser9 occurred concomitantly with cardiomyocyte apoptosis [39]. Moreover, we identified that p-Gsk3 $\beta$ was also dramatically declined in 
miR-155 inhibitors-injected embryos. Collectively, we suggested that knockdown of miR-155 blocked Igf1 pathway and induced apoptosis and may thereby cause cardiac developmental defects in zebrafish embryos.

Serum miRNAs are found to hold promise to serve as novel biomarkers since they are easy to detect and are also stable during sample handling. Recently, several studies detected miRNAs in maternal serum samples which may offer a noninvasive test for monitoring pregnancy-associated diseases. For example, Gu et al [40] revealed six miRNAs that are upregulated in the serum of pregnant women and could be used as biomarkers for the diagnosis of fetal neural tube defects. Mouillet et al [41] analyzed the serum levels of miRNAs of pregnant women with fetuses exhibiting fetal growth restriction (FGR) and determined that FGR is associated with increased miR-517a, $-518 b,-518$ e and -524 levels in maternal serum. In the current study, we detected the serum levels of miR-155 and found that the levels of miR-155 were increased in serum from GDM patients, especially in these GDM patients whose blood glucose was poorly controlled. Besides, maternal serum concentration of miR-155 was positively correlated with the neonatal ventricular walls thicknesses, indicating that serum miR-155 might be a potential biomarker for GDM-induced CHDs.

\section{Conclusions:}

Collectively, the present study demonstrated for the first time that miR-155 is essential for cardiac development. Under high glucose condition, upregulation of miR-155 activated Igf1-Akt-Gsk3 $\beta$ pathway and enhanced the production of ROS and may thereby exert teratogenic effect on cardiac development. Besides, knockdown of miR-155 could block Igf1 pathway and induce apoptosis and may thus cause cardiac developmental defects in zebrafish embryos. Moreover, miR-155 is increased in serum from GDM patients and correlated with fetal ventricular thickness, and it might be a novel diagnostic biomarker as well as a potential drug target of GDM induced-CHDs.

\section{Abbreviations}

GDM: gestational diabetes mellitus; ROS: reactive oxygen species; CHDs: congenital heart defects; RVWT: right ventricular wall thickness; LVWT: left ventricular wall thickness; IVST: interventricular septum thickness; ETS1: Ets proto-oncogene 1; NAC: N-acetylcysteine; DMSO: dimethyl sulphoxide; HPF: hours post-fertilization; BCA: bicinchoninic acid; IGF1: insulin-like growth factor 1; GSK3: glycogen synthase kinase-3ß; MOs: morpholinos; DCFH-DA: 2',7'-Dichlorodihydrofluorescein diacetate; GPX: glutathione peroxidase; BCL2: B celllymphoma/leukemia 2gene; AKT: alpha serine/threonine-protein kinase; BAX: baxdelta2omega ; PVDF: polyvinylidene difluoride.

\section{Declarations}

Acknowledgments 
None.

\section{Authors' contributions}

LY, ZXT conceived and designed the experiments; KZ, YJZ, XJY, BJ performed the qPCR, western blot, ROS detection and examined the serum miR-155; MYH, LYW, DLL constructed the plasmids and conducted micro-injection; YJZ, XJY, YZ, HJL monitored zebrafish embryos development and took the photo. KZ, LTW, XJW analyzed and interpreted the data; KZ, BJ, LY, ZXT wrote the paper; all authors read and approved the final manuscript.

\section{Funding}

This work was supported by grants from: (1) the Youth Program of National Natural Science Foundation of China (81200125) to LY; (2) the Priority Academic Program Development (PAPD) and the Key Project of Natural Science Foundation of the Higher Education Institutions (17KJA310001) to LY; (3) the Six Talent Peaks Project of Jiangsu Province (2015-WSN-032) to ZXT.

\section{Availability of data and materials}

All data and materials are available upon request

\section{Ethics approval and consent to participate}

All experiments were approved by the medical ethics committee of Nanjing Medical University and the ethics committee of the Jiangsu province Hospital.

\section{Consent for publication}

All authors have declared their consent for this publication.

\section{Competing interests}

The authors declare that they have no competing interests.

\section{References}

1. Øyen N, Diaz LJ, Leirgul E, Boyd HA, Priest J, Mathiesen ER, Quertermous T, Wohlfahrt J, Melbye M: Prepregnancy Diabetes and Offspring Risk of Congenital Heart Disease: A Nationwide Cohort Study. Circulation 2016, 133(23):2243-2253.

2. Correa A: Pregestational Diabetes Mellitus and Congenital Heart Defects. Circulation 2016, 133(23):2219-2221.

3. Engineer A, Saiyin T, Lu X, Kucey AS, Urquhart BL, Drysdale TA, Norozi K, Feng Q: Sapropterin Treatment Prevents Congenital Heart Defects Induced by Pregestational Diabetes Mellitus in Mice. $J$ Am Heart Assoc 2018, 7(21):e009624. 
4. Liang J, Gui Y, Wang W, Gao S, Li J, Song H: Elevated glucose induces congenital heart defects by altering the expression of tbx5, tbx20, and has2 in developing zebrafish embryos. Birth Defects Res $A$ Clin Mol Teratol 2010, 88(6):480-486.

5. Michaille JJ, Awad H, Fortman EC, Efanov AA, Tili E: miR-155 expression in antitumor immunity: The higher the better. Genes Chromosomes Cancer 2019, 58(4):208-218.

6. Li N, Cui T, Guo W, Wang D, Mao L: MiR-155-5p accelerates the metastasis of cervical cancer cell via targeting TP53INP1. Onco Targets Ther 2019, 12:3181-3196.

7. Burocchi A, Pittoni P, Tili E, Rigoni A, Costinean S, Croce CM, Colombo MP: Regulated Expression of miR-155 is Required for iNKT Cell Development. Front Immunol 2015, 6:140.

8. Zhang D, Cui Y, Li B, Luo X, Li B, Tang Y: miR-155 regulates high glucose-induced cardiac fibrosis via the TGF- $\beta$ signaling pathway. Mol Biosyst 2016, 13(1):215-224.

9. Gao Z, Kim GH, Mackinnon AC, Flagg AE, Bassett B, Earley JU, Svensson EC: Ets1 is required for proper migration and differentiation of the cardiac neural crest. Development 2010, 137(9):15431551.

10. Lie-Venema H, Gittenberger-de GAC, van Empel LJ, Boot MJ, Kerkdijk H, de Kant E, DeRuiter MC: Ets-1 and Ets-2 transcription factors are essential for normal coronary and myocardial development in chicken embryos. Circ Res 2003, 92(7):749-756.

11. Wander PL, Boyko EJ, Hevner K, Parikh VJ, Tadesse MG, Sorensen TK, Williams MA, Enquobahrie DA: Circulating early- and mid-pregnancy microRNAs and risk of gestational diabetes. Diabetes Res Clin Pract 2017, 132:1-9.

12. Giardoglou P, Beis D. On Zebrafish Disease Models and Matters of the Heart. Biomedicines. 2019. 7(1).

13. Staudt D, Stainier D. Uncovering the molecular and cellular mechanisms of heart development using the zebrafish. Annu Rev Genet. 2012. 46: 397-418.

14. Heckler K, Kroll J. Zebrafish as a Model for the Study of Microvascular Complications of Diabetes and Their Mechanisms. Int J Mol Sci. 2017. 18(9).

15. Singh A, Castillo HA, Brown J, Kaslin J, Dwyer KM, Gibert Y: High glucose levels affect retinal patterning during zebrafish embryogenesis. Sci Rep 2019, 9(1):4121.

16. Gleeson M, Connaughton V, Arneson LS: Induction of hyperglycaemia in zebrafish (Danio rerio) leads to morphological changes in the retina. Acta Diabeto/ 2007, 44(3):157-163.

17. Metzger BE, Lowe LP, Dyer AR, et al. Hyperglycemia and adverse pregnancy outcomes. N Engl J Med. 2008. 358(19): 1991-2002.

18. Sun Y, Wang Q, Fang Y, Wu C, Lu G, Chen Z: Activation of the Nkx2.5-Calr-p53 signaling pathway by hyperglycemia induces cardiac remodeling and dysfunction in adult zebrafish. Dis Model Mech 2017, 10(10):1217-1227.

19. Kloosterman WP, Steiner FA, Berezikov E, et al. Cloning and expression of new microRNAs from zebrafish. Nucleic Acids Res. 2006. 34(9): 2558-69. 
20. Alcaraz-Pérez F, Mulero V, Cayuela ML. Application of the dual-luciferase reporter assay to the analysis of promoter activity in Zebrafish embryos. BMC Biotechnol. 2008. 8: 81.

21. Hyatt TM, Ekker SC. Vectors and techniques for ectopic gene expression in zebrafish. Methods Cell Biol. 1999. 59: 117-26.

22. Hu R, Huffaker TB, Kagele DA, et al. MicroRNA-155 confers encephalogenic potential to Th17 cells by promoting effector gene expression. J Immunol. 2013. 190(12): 5972-80.

23. Seimi SK, Seinosuke K, Tsuyoshi S, Tomomi U, Tetsuaki H, Miki K, Ryuji T, Kenji I, Mitsuhiro Y: Glycogen synthase kinase-3beta is involved in the process of myocardial hypertrophy stimulated by insulin-like growth factor-1. Circ J 2004, 68(3):247-253.

24. Kobayashi S, Nakamura TY, Wakabayashi S: Calcineurin B homologous protein 3 negatively regulates cardiomyocyte hypertrophy via inhibition of glycogen synthase kinase 3 phosphorylation. $J$ Mol Cell Cardiol 2015, 84:133-142.

25. Onodera Y, Teramura T, Takehara T, Obora K, Mori T, Fukuda K. miR-155 induces ROS generation through downregulation of antioxidation-related genes in mesenchymal stem cells. Aging Cell. 2017. 16(6): 1369-1380.

26. Razaghi B, Steele SL, Prykhozhij SV, et al. hace1 Influences zebrafish cardiac development via ROSdependent mechanisms. Dev Dyn. 2018. 247(2): 289-303.

27. Nasr-Esfahani MH, Aitken JR, Johnson MH. Hydrogen peroxide levels in mouse oocytes and early cleavage stage embryos developed in vitro or in vivo. Development. 1990. 109(2): 501-7.

28. Peruzzi F, Prisco M, Dews M, et al. Multiple signaling pathways of the insulin-like growth factor 1 receptor in protection from apoptosis. Mol Cell Biol. 1999. 19(10): 7203-15.

29. Kang BP, Urbonas A, Baddoo A, Baskin S, Malhotra A, Meggs LG. IGF-1 inhibits the mitochondrial apoptosis program in mesangial cells exposed to high glucose. Am J Physiol Renal Physiol. 2003. 285(5): F1013-24.

30. Bouma BJ, Mulder BJ. Changing Landscape of Congenital Heart Disease. Circ Res. 2017. 120(6): 908-922.

31. Garcia-Vargas L, Addison SS, Nistala R, Kurukulasuriya D, Sowers JR: Gestational Diabetes and the Offspring: Implications in the Development of the Cardiorenal Metabolic Syndrome in Offspring. Cardiorenal Med 2012, 2(2):134-142.

32. Fine EL, Horal M, Chang TI, Fortin G, Loeken MR: Evidence that elevated glucose causes altered gene expression, apoptosis, and neural tube defects in a mouse model of diabetic pregnancy. Diabetes 1999, 48(12):2454-2462.

33. Arow M, Waldman M, Yadin D, Nudelman V, Shainberg A, Abraham NG, Freimark D, Kornowski R, Aravot D, Hochhauser E et al: Sodium-glucose cotransporter 2 inhibitor Dapagliflozin attenuates diabetic cardiomyopathy. Cardiovasc Diabetol 2020, 19(1):7.

34. La Sala L, Mrakic-Sposta S, Micheloni S, Prattichizzo F, Ceriello A: Glucose-sensing microRNA-21 disrupts ROS homeostasis and impairs antioxidant responses in cellular glucose variability. Cardiovasc Diabetol 2018, 17(1):105. 
35. Verschoor ML, Verschoor CP, Singh G: Ets-1 global gene expression profile reveals associations with metabolism and oxidative stress in ovarian and breast cancers. Cancer Metab 2013, 1(1):17.

36. Drexler AL, Pietri JE, Pakpour N, et al. Human IGF1 regulates midgut oxidative stress and epithelial homeostasis to balance lifespan and Plasmodium falciparum resistance in Anopheles stephensi. PLoS Pathog. 2014. 10(6): e1004231.

37. llatovskaya DV, Pavlov TS, Levchenko V, Staruschenko A. ROS production as a common mechanism of ENaC regulation by EGF, insulin, and IGF-1. Am J Physiol Cell Physiol. 2013. 304(1): C102-11.

38. Ren J, Samson WK, Sowers JR: Insulin-like growth factor I as a cardiac hormone: physiological and pathophysiological implications in heart disease. J Mol Cell Cardiol 1999, 31(11):2049-2061.

39. Su D, Zhao J, Hu S, Guan L, Li Q, Shi C, Ma X, Gou J, Zhou Y: GSK3 $\beta$ and MCL-1 mediate cardiomyocyte apoptosis in response to high glucose. Histochem Cell Biol 2019, 152(3):217-225.

40. Gu H, Li H, Zhang L, Luan H, Huang T, Wang L, Fan Y, Zhang Y, Liu X, Wang W et al: Diagnostic role of microRNA expression profile in the serum of pregnant women with fetuses with neural tube defects. J Neurochem 2012, 122(3):641-649.

41. Mouillet JF, Chu T, Hubel CA, Nelson DM, Parks WT, Sadovsky Y: The levels of hypoxia-regulated microRNAs in plasma of pregnant women with fetal growth restriction. Placenta 2010, 31(9):781784.

\section{Figures}


a

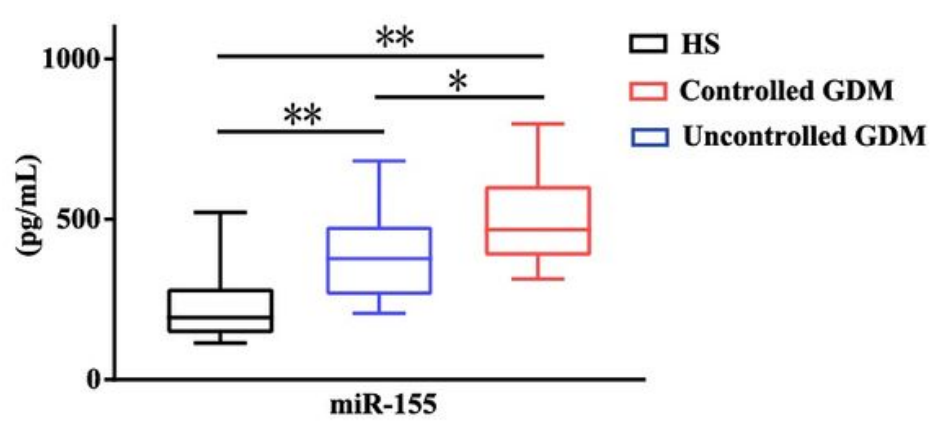

c

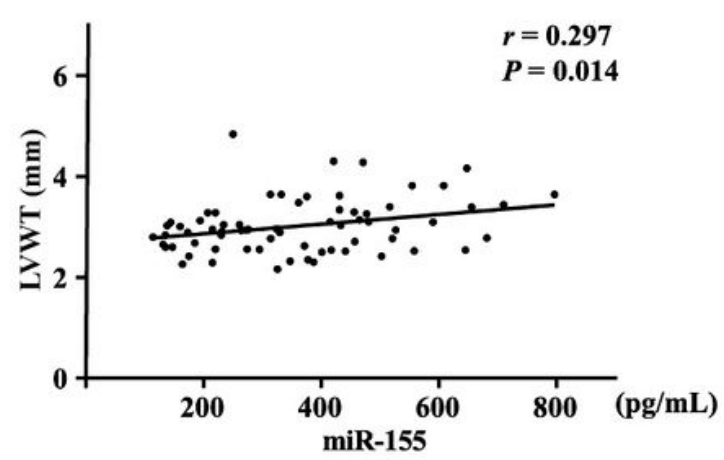

e

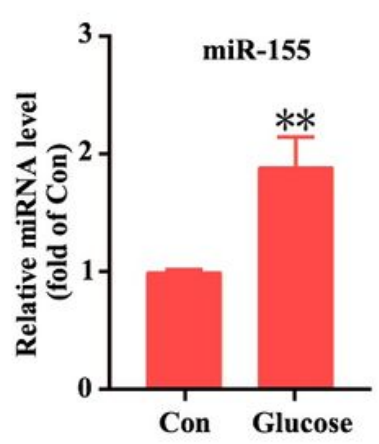

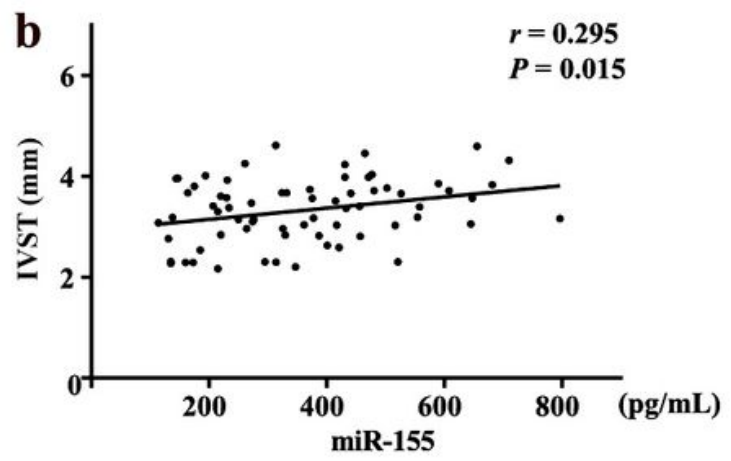

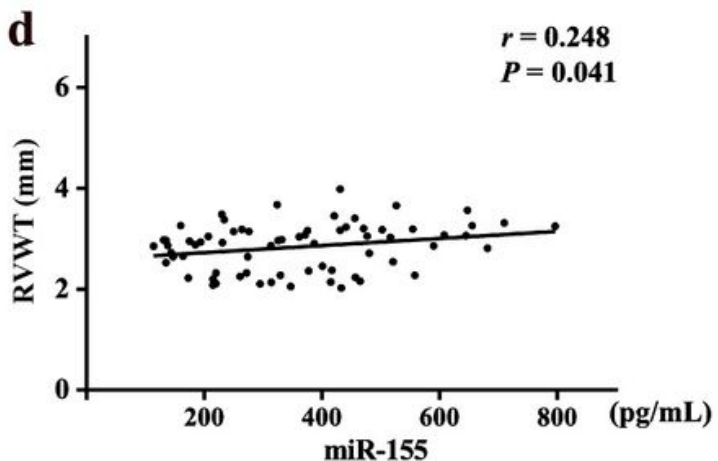

$\mathbf{f}$ 72 hpf
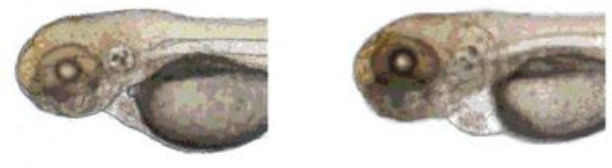

Glucose
Glucose+ miR-155 inhibitor g

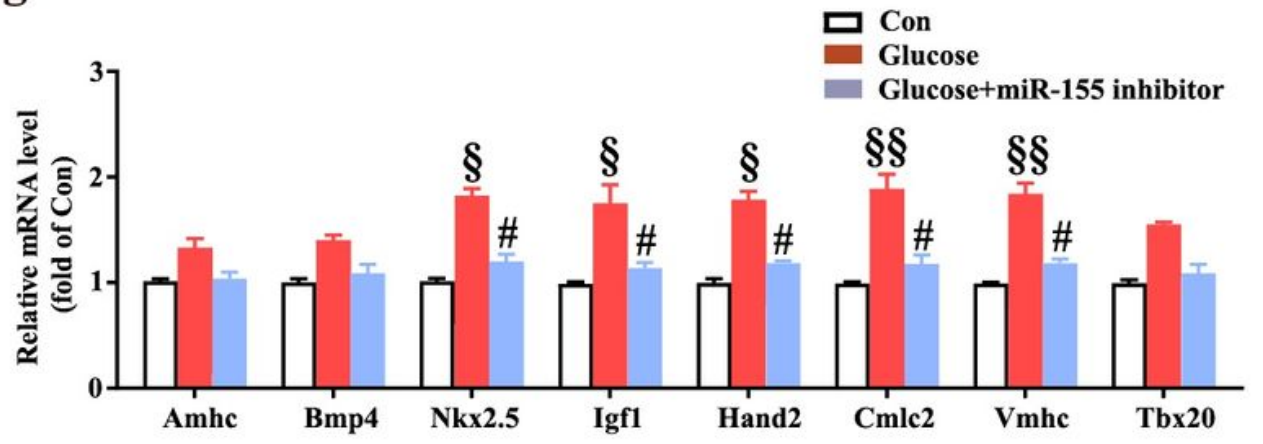

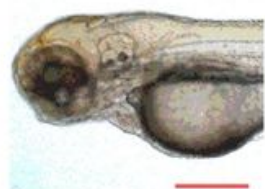

h

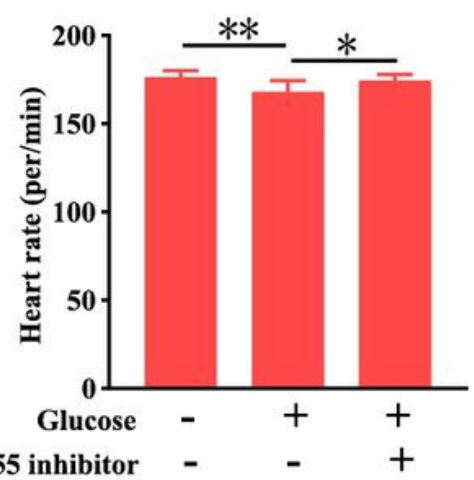

Figure 1

Upregulation of miR-155 is correlated with high glucose-induced cardiac developmental defects. a Maternal serum levels of miR-155 in healthy subjects (HS) $(n=25)$, controlled groups $(n=23)$ and uncontrolled groups were detected by ELISA $(n=20)$. b-d Maternal serum levels of miR-155 are positively associated with fetal IVST ( $r=0.295, P \llbracket 0.05)$, LVWT ( $r=0.297, P \otimes 0.05)$ and RVWT ( $r=0.248, P \otimes 0.05)$. e The expression levels of miR-155 in high glucose-treated zebrafish embryos at $24 \mathrm{hpf}$ were analyzed by qRTPCR. f Zebrafish embryos were exposed to high glucose. In rescue experiments, zebrafish embryos were 
injected with $3 \mu \mathrm{M}$ of miR-155 inhibitors. Bars, $250 \mu \mathrm{m}$. g Cardiac specific markers mRNA levels at $72 \mathrm{hpf}$ were analyzed by qRT-PCR. h Heart rate of zebrafish embryos were determined at $72 \mathrm{hpf}$. Data were presented as the mean $\pm S D$ of three individual experiments. ${ }^{*} P<0.05,{ }^{*} \mathrm{P}<0.01 . \S \mathrm{P}<0.05, \S \S \mathrm{P}<0.01$ vs corresponding control groups. \#P<0.05 vs corresponding high glucose-treated groups.

a

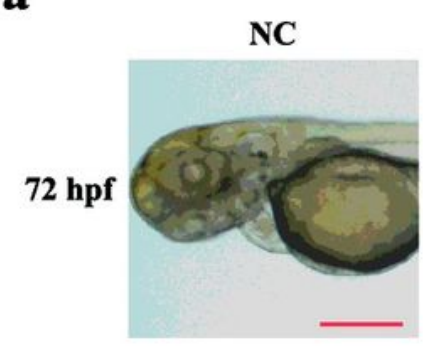

b
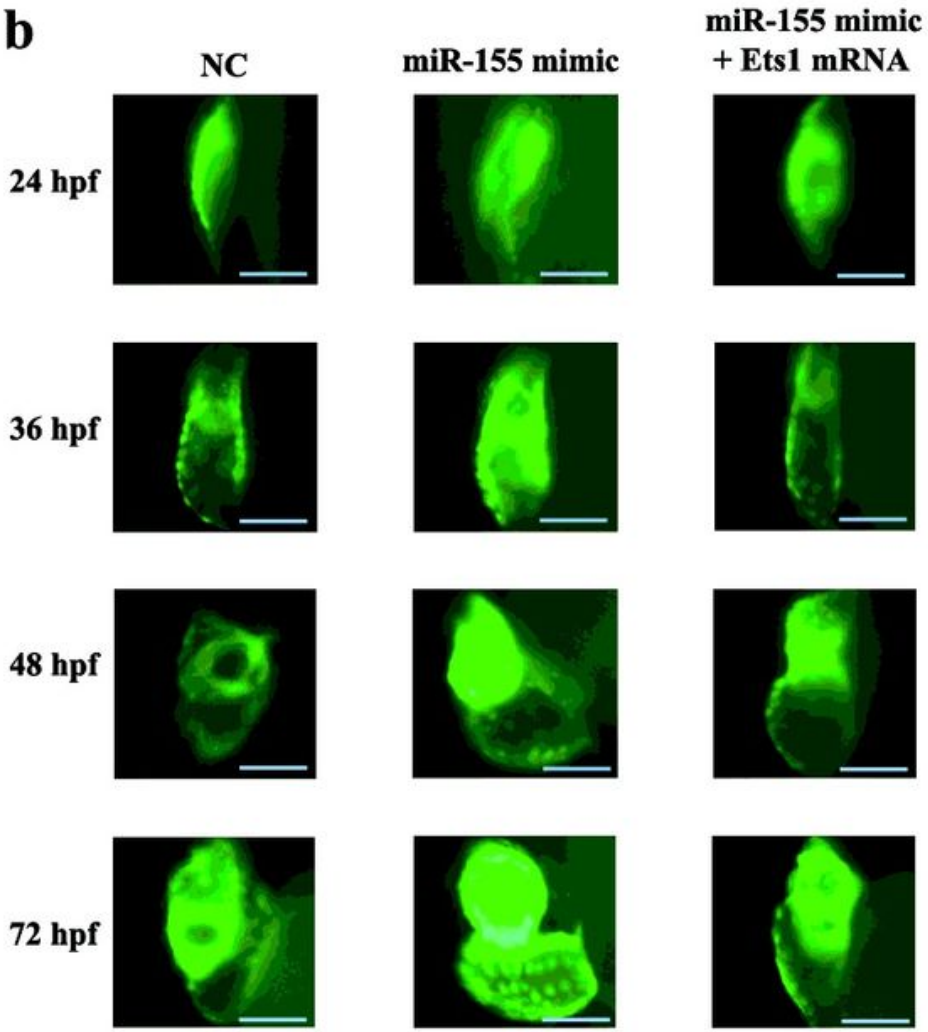

d

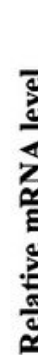

(3)
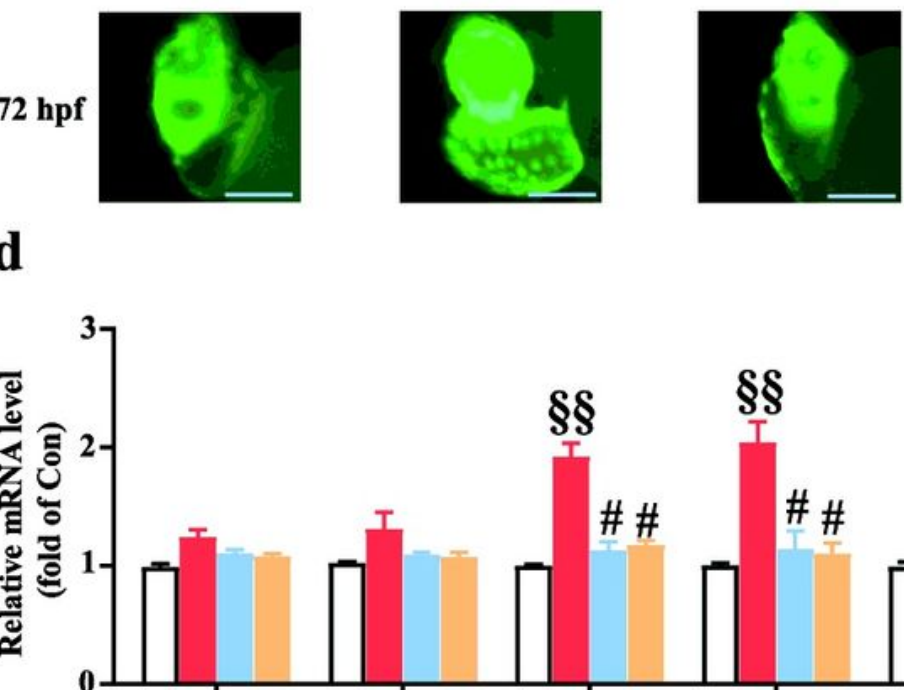

miR-155 mimic + miR-155 inhibitor

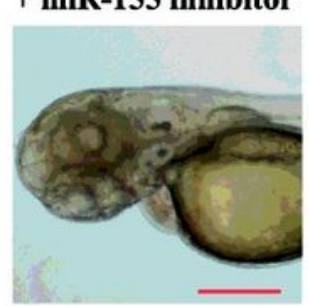

miR-155 mimic + Ets1 mRNA

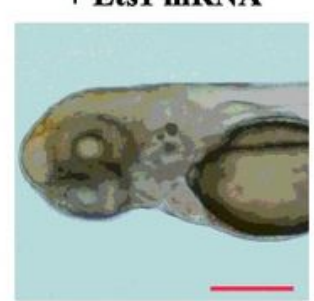

C

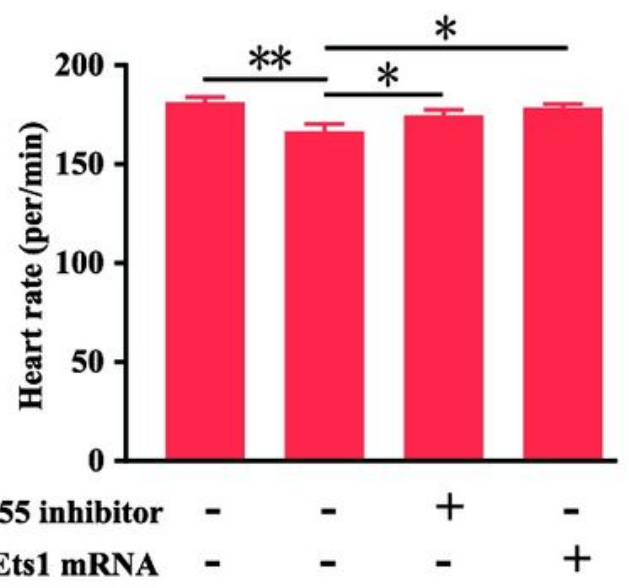

Figure 2
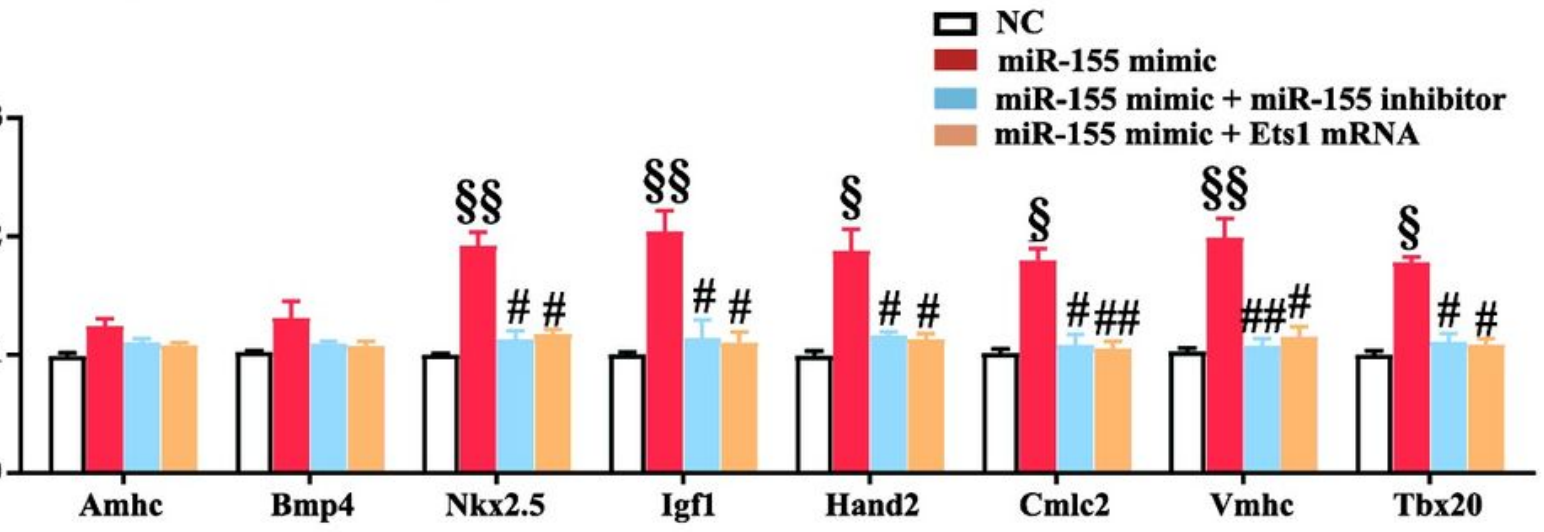
Overexpression of miR-155 causes zebrafish cardiac developmental defects. a Zebrafish embryos were injected with $3 \mu \mathrm{M}$ of miR-155 mimics. In rescue experiments, $3 \mu \mathrm{M}$ of miR-155 mimics were injected together with $3 \mu \mathrm{M}$ of miR-155 inhibitors or $30 \mathrm{pg}$ of Ets1 mRNA. Bars, $250 \mu \mathrm{m}$. b Cmlc2:GFP zebrafish embryos were injected with $3 \mu \mathrm{M}$ of miR-155mimics. In rescue experiments, $3 \mu \mathrm{M}$ of miR-155 mimics injected together with $30 \mathrm{pg}$ of Ets $1 \mathrm{mRNA}$. Bars, $100 \mu \mathrm{m}$. c Heart rate of zebrafish embryos was determined at $72 \mathrm{hpf}$. $\mathrm{d}$ Cardiac specific markers mRNA levels at $72 \mathrm{hpf}$ were analyzed by qRT-PCR. Data were presented as the mean $\pm S D$ of three individual experiments. ${ }^{*} P<0.05,{ }^{*} P<0.01 . \S P<0.05, \S \S P<0.01$ vs corresponding NC groups. \#P<0.05, \#\#P<0.01 vs corresponding miR-155 mimics-injected groups.

a
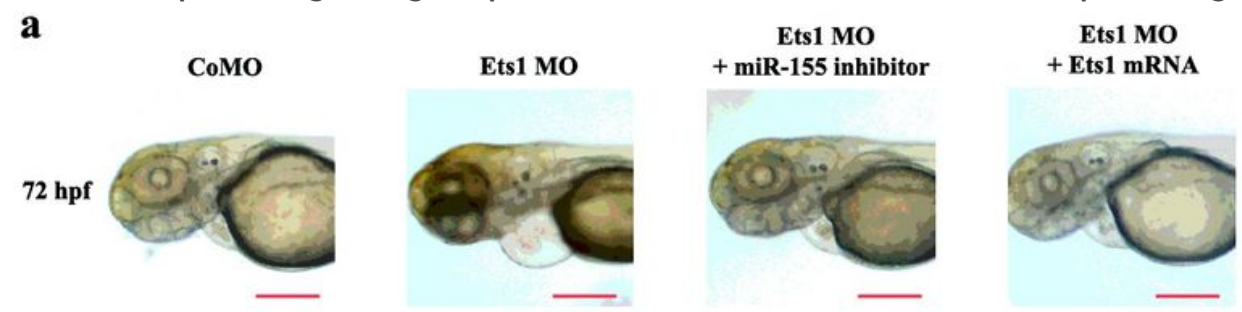

c

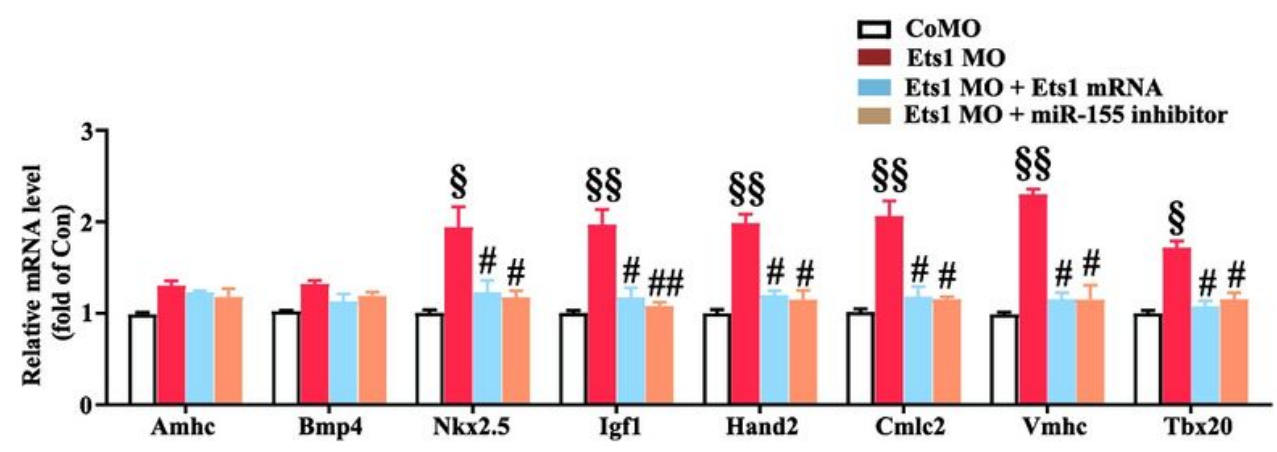

b

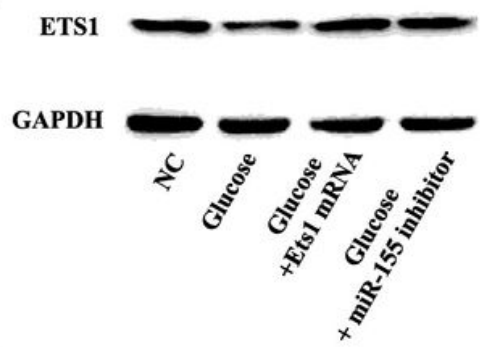

d

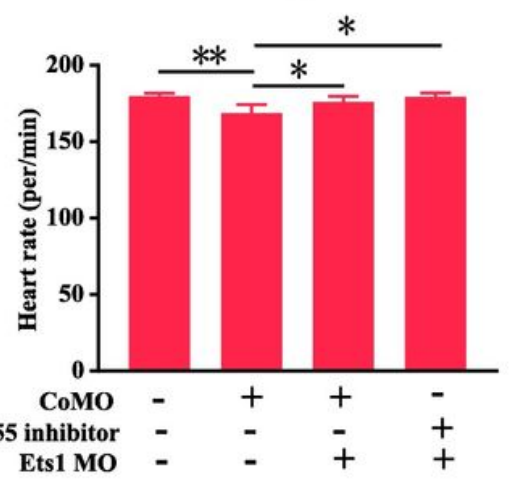

Figure 3

miR-155 regulates zebrafish cardiac development by targeting Ets 1 . a Zebrafish embryos were injected with $5 \mathrm{ng}$ of Ets $1 \mathrm{MO}$. In rescue experiments, $5 \mathrm{ng}$ of Ets $1 \mathrm{MO}$ were injected together with $3 \mu \mathrm{M}$ of miR155 inhibitors or $30 \mathrm{pg}$ of Ets1 mRNA. Bars, $250 \mu \mathrm{m}$. b Zebrafish embryos were treated with high glucose. In rescue experiments, zebrafish embryos were injected with $30 \mathrm{pg}$ of Ets 1 mRNA or $3 \mu \mathrm{M}$ of miR-155 inhibitors. Protein levels of Ets 1 at $36 \mathrm{hpf}$ were analyzed by western blot analysis. c Cardiac specific markers mRNA levels at $72 \mathrm{hpf}$ were analyzed by qRT-PCR. $\mathrm{d}$ Heart rate of zebrafish embryos were determined at $72 \mathrm{hpf}$. Data were presented as the mean \pm SD of three individual experiments. ${ }^{*} P<0.05$, ${ }^{\star *} \mathrm{P}<0.01$. $\S \mathrm{P}<0.05, \S \S \mathrm{P}<0.01$ vs corresponding control groups. $\# \mathrm{P}<0.05$, \#\#P<0.01 vs corresponding Ets 1 MO-injected groups. 
a

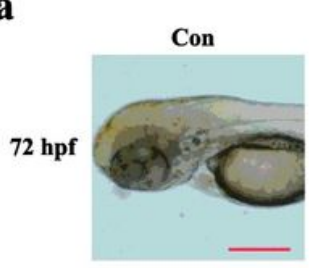

b

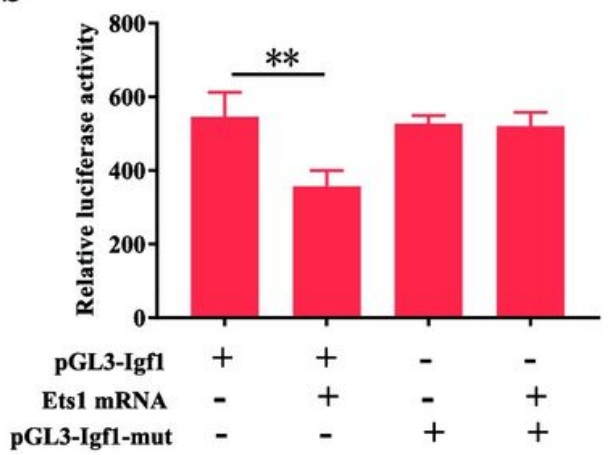

Igf1 mRNA

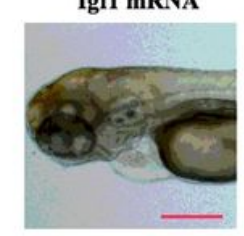

c
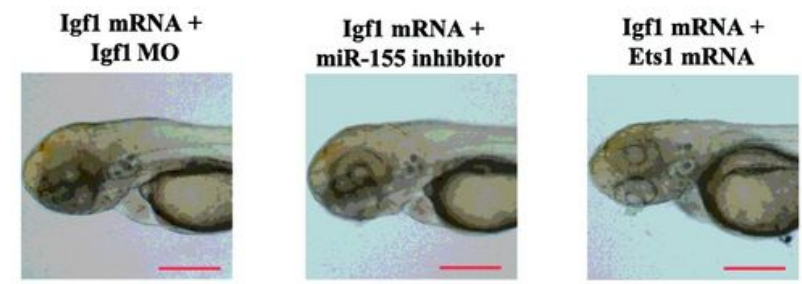

e

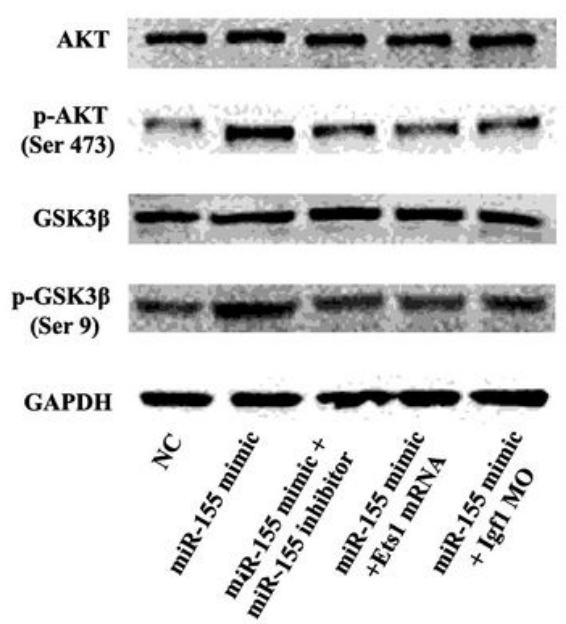

f
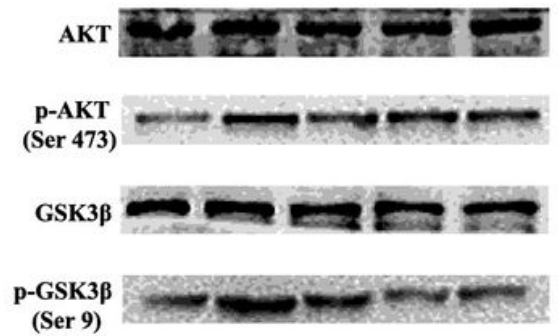

GAPDH

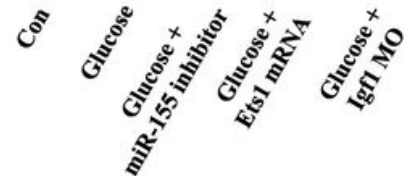

Figure 4

Upregulation of miR-155 activated Igf1 pathway in zebrafish embryos. a Zebrafish embryos were injected with $100 \mathrm{pg}$ of Igf1 mRNA. In rescue experiments, 100 pg of Igf1 mRNA were injected together with 3 ng of Igf1 $\mathrm{MO}$ or $3 \mu \mathrm{M}$ of miR-155 inhibitors or 30 pg of Ets1 mRNA. Bars, $250 \mu \mathrm{m}$. b Luciferase activity was analyzed following co-transfection of zebrafish embryos with pGL3-basic-zebrafish-Igf1-promoter, pGL3mutant-zebrafish-Igf1-promoter and Ets1 mRNA. c Heart rates of zebrafish embryos were determined at 72 hpf. d Cardiac specific markers mRNA levels at 72 hpf were analyzed by qRT-PCR. $§ P<0.05, \S \S P<0.01$ vs corresponding control groups. \#P<0.05, \#\#P<0.01 vs corresponding Igf1 mRNA-injected groups. e,g Zebrafish embryos were injected with $3 \mu \mathrm{M}$ of miR-155 mimics, or $3 \mu \mathrm{M}$ of miR-155 mimics were injected 
together with $3 \mu \mathrm{M}$ of miR-155 inhibitors or $30 \mathrm{pg}$ of Ets 1 mRNA or $3 \mathrm{ng}$ of Igf MO. Protein levels of Akt, pAkt, Gsk3ß, p-Gsk3 $\beta$ at $36 \mathrm{hpf}$ were analyzed by western blot analysis. $\S \mathrm{P}<0.05, \S \S \mathrm{P}<0.01 \mathrm{vs}$ corresponding control groups. \#P<0.05, \#\#P<0.01 vs corresponding miR-155 mimics-injected groups. f,h Zebrafish embryos were exposed to $2 \% \mathrm{D}$-glucose. In rescue experiments, zebrafish embryos were injected with $3 \mu \mathrm{M}$ of miR-155 inhibitors or $30 \mathrm{pg}$ of Ets 1 mRNA or $3 \mathrm{ng}$ of Igf MO. Protein levels of Akt, pAkt, Gsk3ß, p-Gsk3ß at $36 \mathrm{hpf}$ were analyzed by western blot analysis. $\S \mathrm{P}<0.05, \S \S \mathrm{P}<0.01$ vs corresponding control groups. \#P<0.05, \#\#P<0.01 vs corresponding high glucose-treated groups. Data were presented as the mean \pm SD of three individual experiments.

a
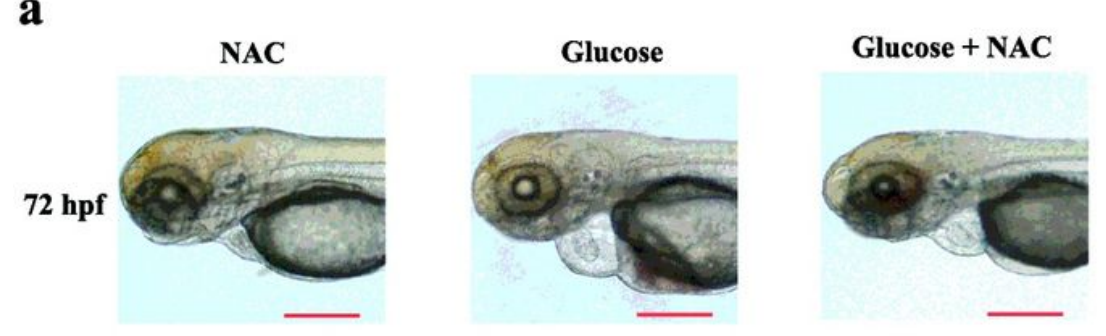

c

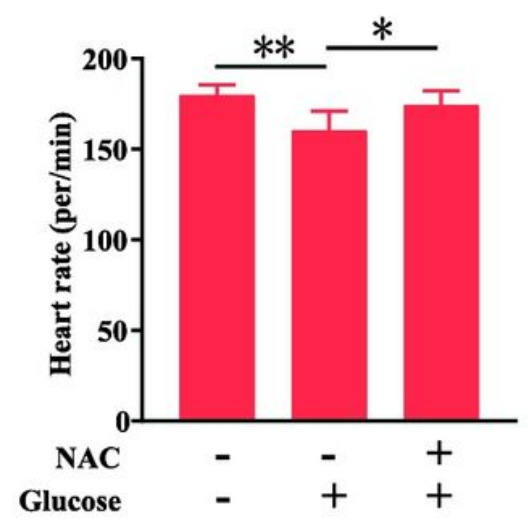

f
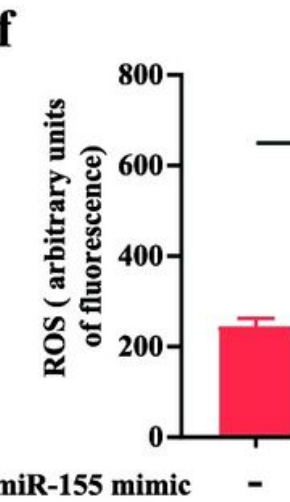

d

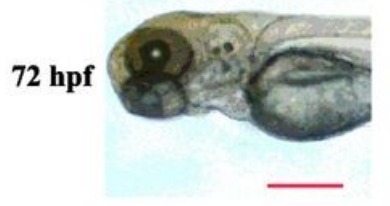

b
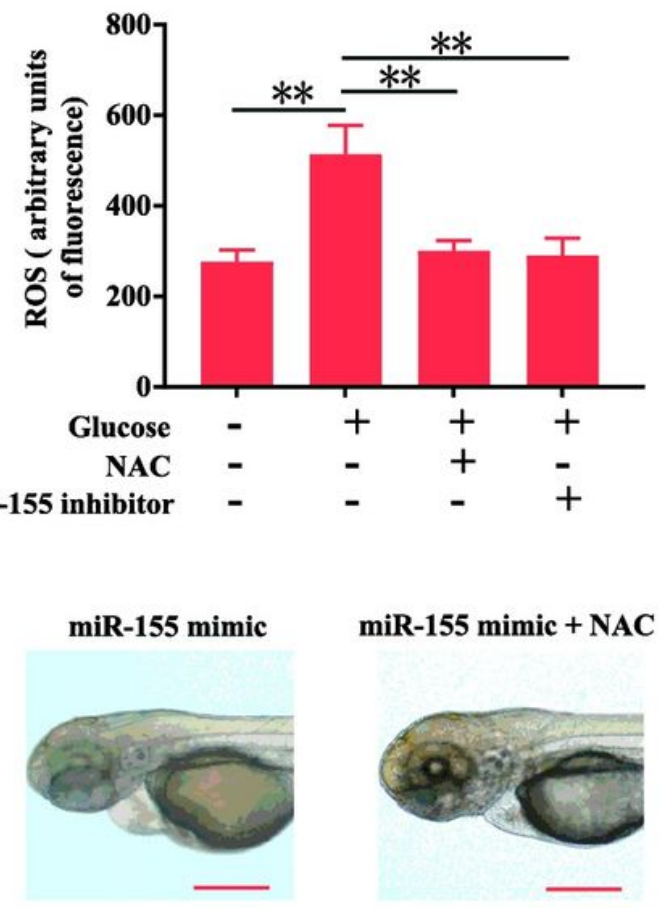

g

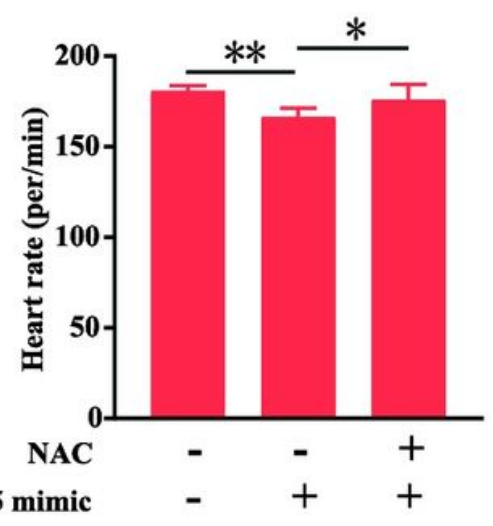

\section{Figure 5}

Upregulation of miR-155 elevated the production of ROS in zebrafish embryos. a Zebrafish embryos were treated with high glucose. In rescue experiments, $100 \mu \mathrm{M}$ of NAC were treated. Bars, $250 \mu \mathrm{m}$. b Reactive 
oxygen species production was monitored in the zebrafish embryos treated with high glucose and $3 \mu \mathrm{M}$ of miR-155 inhibitor or $100 \mu \mathrm{M}$ of NAC. c,g Heart rates of zebrafish embryos were determined at $72 \mathrm{hpf}$. $\mathrm{d}$ Zebrafish embryos were treated with $3 \mu \mathrm{M}$ of miR-155 mimics. In rescue experiments, $100 \mu \mathrm{M}$ of NAC were treated. Bars, $250 \mu \mathrm{m}$. e The expression of miR-155 in NAC-treated zebrafish embryos were analyzed by qRT-PCR. $f$ Reactive oxygen species production was monitored in the zebrafish embryos treated with 3 $\mu \mathrm{M}$ of miR-155 mimics and $100 \mu \mathrm{M}$ of NAC or $30 \mathrm{pg}$ of Ets 1 mRNA or $3 \mathrm{ng}$ of Igf $1 \mathrm{MO}$. Data were presented as the mean $\pm S D$ of three individual experiments. ${ }^{*} P<0.05, * * P<0.01$.

a

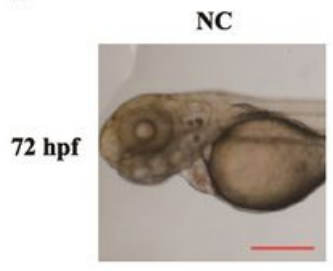

miR-155 inhibitor

+ miR-155 mimic

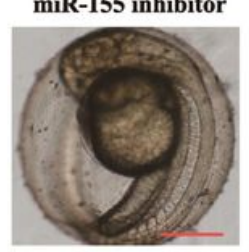

48 hpf
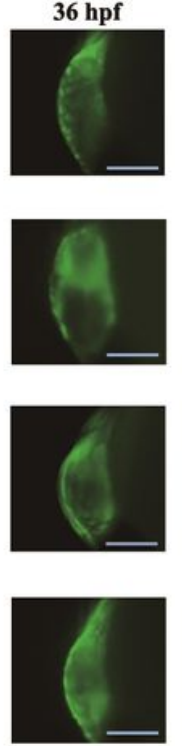

+ Igf1 mRNA

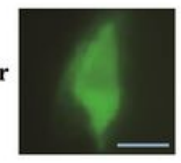

d

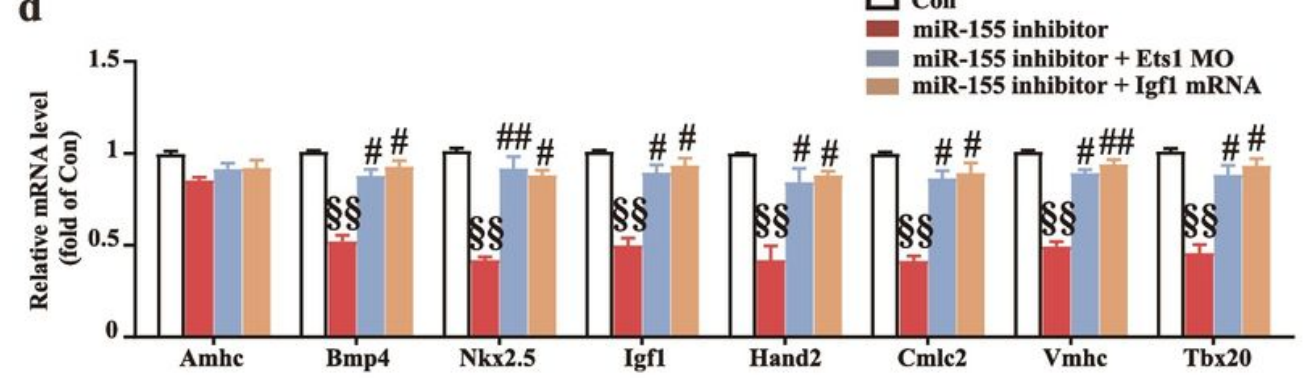

miR-155 inhibitor

+ Ets1 MO

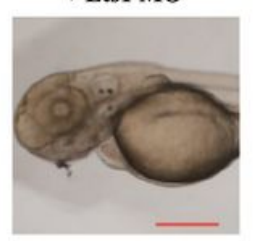

c

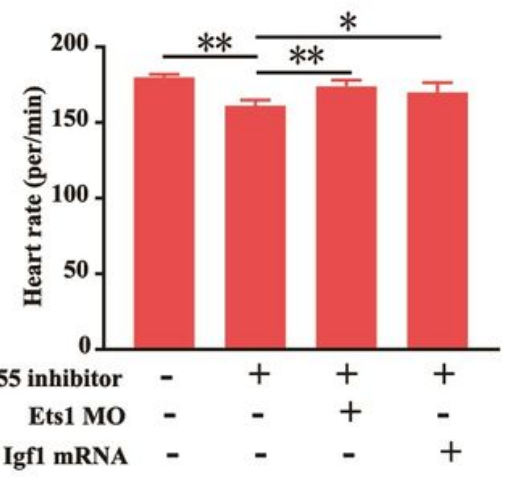

$\mathbf{e}$
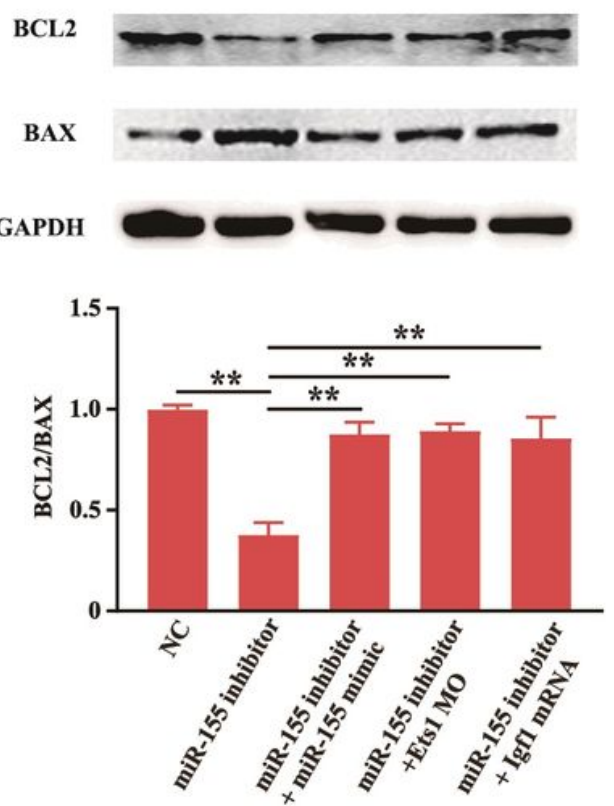

\section{Figure 6}

Knockdown of miR-155 can also induce cardiac developmental defects in zebrafish embryos. a Zebrafish embryos were injected with $5 \mu \mathrm{M}$ of miR-155 inhibitors. In rescue experiments, $5 \mu \mathrm{M}$ of miR-155 inhibitors were injected together with $5 \mu \mathrm{M}$ of miR-155 mimics or $7.5 \mathrm{ng}$ of Ets $1 \mathrm{MO}$ or $150 \mathrm{pg}$ of Igf $1 \mathrm{mRNA}$. Bars, 
$250 \mu \mathrm{m}$. b Cmlc2:GFP zebrafish embryos were injected with $5 \mu \mathrm{M}$ of miR-155 inhibitors or $5 \mu \mathrm{M}$ of miR155 inhibitors injected together with $7.5 \mathrm{ng}$ of Ets $1 \mathrm{MO}$ or $150 \mathrm{pg}$ of Igf1 mRNA. Bars, $100 \mu \mathrm{m}$. c Heart rate of zebrafish embryos were determined at $72 \mathrm{hpf}$. d Cardiac specific markers mRNA levels at $72 \mathrm{hpf}$ were analyzed by qRT-PCR. e Zebrafish embryos were injected with $5 \mu \mathrm{M}$ of miR-155 inhibitors or $5 \mu \mathrm{M}$ of miR155 inhibitors were injected together with $5 \mu \mathrm{M}$ of miR-155 mimics or $7.5 \mathrm{ng}$ of Ets $1 \mathrm{MO}$ or $150 \mathrm{pg}$ of Igf1 mRNA. Protein levels of $\mathrm{Bcl} 2$ and $\mathrm{Bax}$ at $36 \mathrm{hpf}$ were analyzed by western blot analysis. Data were presented as the mean $\pm S D$ of three individual experiments. ${ }^{*} P<0.05,{ }^{*} \mathrm{P}<0.01 . \S P<0.05, \S \S P<0.01$ vs corresponding con groups. $\# \mathrm{P}<0.05, \# \# \mathrm{P}<0.01$ vs corresponding miR-155 inhibitor-injected groups.

\section{Supplementary Files}

This is a list of supplementary files associated with this preprint. Click to download.

- Supplementarymaterial.docx 